\title{
The Phase Structure of Antiferromagnetic Ising Model in the Presence of Frustrations
}

\author{
A.Z. Akheyan*,N.S. Ananikian ${ }^{\dagger}$, S.K. Dallakian ${ }^{\ddagger}$ \\ Department of Theoretical Physics, Yerevan Physics Institute, \\ Alikhanian Br.2, 375036 Yerevan, Armenia
}

July 23, 2021

\begin{abstract}
The antiferromagnetic Ising model in a magnetic field is considered on the Husimi tree. Using iteration technique we draw the plots of magnetization versus external field for different temperatures and construct the resulting phase diagram. We show that frustration effects essentially change the critical properties. The model exhibits three distinct critical regions, including re-entrant phase structure and Griffiths singularities.
\end{abstract}

*e-mail: akheyan@lx2.yerphi.am

${ }^{\dagger}$ e-mail: ananik@jerewan1.yerphi.am

‡e-mail: saco@atlas.yerphi.am 
It is now widely recognized that Bethe-like approximations are more reliable than conventional mean-field approximation. In mean-field approximation one replaces the interaction of a given site with the rest of a system by a mean field and thus completely neglects fluctuations. In Bethe approximation one first calculates the fluctuations in a microscopic region and only then replaces the interaction to the rest of a system by a mean field. For ferromagnetic and unfrustrated antiferromagnetic models these both approaches provide the same qualitative picture, since both have the same symmetries and large scale behavior. However in models with frustrations the microscopic fluctuations along a loop with even number of bond play important role and essentially change critical picture. Such systems attract now great attention from both experimental and theoretical point of view. The most common example of these models are triangular antiferromagnets, which were reviewed in detail in [1, 2].

It is well known that instead of Bethe-like approximation one can consider exact solution of the models on Bethe or Bethe like hierarchical lattices. To obtain frustration effect we use so called Husimi tree, which is in fact the Bethe lattice constructed from triangles (fig. 1). We observe the most simple Ising model on Husimi tree defined by Hamiltonian:

$$
-H / k T=J \sum_{(i, j)} \sigma_{i} \sigma_{j}+h \sum_{i} \sigma_{i}
$$

with antiferromagnetic coupling $(J<0)$. We have constructed the plots of magnetization versus external field for different temperature and drawn the resulting phase diagram. Due to frustration effects this diagram essentially differs from the one obtained on Bethe lattice. Moreover we observed new critical region which, to our knowledge, has never been described in literature.

Husimi tree can be built successively by attaching $\gamma$ new triangles to each free site of a previous shell. According to this construction one can present the site magnetization $m=\langle\sigma\rangle$ by equation[3]:

$$
m=\frac{e^{2 h} x^{\gamma}-1}{e^{2 h} x^{\gamma}+1},
$$

where $x$ is obtained from the attractor of the following map:

$$
x_{n}=f\left(x_{n-1}\right), \quad f(x)=\frac{e^{4(h+J)} x^{2(\gamma-1)}+2 e^{2 h} x^{\gamma-1}+1}{e^{4 h} x^{2(\gamma-1)}+2 e^{2 h} x^{\gamma-1}+e^{4 J}},
$$

The exact recursion formula allows us to study in detail the critical properties. In thermodynamic limit $(n \rightarrow \infty)$ the recursion sequence $\left\{x_{n}\right\}$ either converges to the stable fixed point $x^{*}$ or to the stable 2 -cycle $\left(x_{1}, x_{2}\right)$. First case corresponds to the phase without sublattice structure, while in second case we have the antiferromagnetic ordering where the values $m_{1}$ and $m_{2}$ are the magnetizations of two sublattices 4 . Proceeding from these principles, we can now construct the plots of magnetization versus external field for different temperatures. 
In fig. 2 we present the plot of $m$ versus $h$ at low temperature. This graphic is typical for antiferromagnetic models at low temperature and is the result of competition between first and second term in Hamiltonian (11). The plots of magnetization for higher temperatures are shown in fig.3,4, and resulting phase diagram is shown in fig.5.

The graphics like shown in fig. 3 are completely absent in antiferromagnetic Ising model on Bethe lattice, and its appearance here is due to frustrations on triangles.

The above result can be explained by considering the frustration as a background disorder in the sense that if two spins on triangle aligned antiparallel then the average value of magnetization on the third site is zero in the absence of magnetic field at all temperatures. Note that the background disorder due to frustration disappears when we introduce the magnetic field.

Thus when we increase the temperature, the background disorder makes spontaneous magnetization zero at lower temperature $T_{1}$ than it should be in unfrustrated case, and, as a result, one obtains the dependence of magnetization from magnetic field shown in figure 3. This eight-like structure survives until reaching to another temperature $T_{2}$. Note that at all range of temperatures between $T_{1}$ and $T_{2}$ we have continuous phase transitions in zero magnetic field.

As we continue to increase temperature the non zero magnetic field is required to overcome the collective effects of frustration and thermal fluctuations, and for temperatures higher than $T_{3}$ we have complete disorder.

Though to our knowledge the obtained phase diagram has not been presented in literature before, it has a number of confirmations with experimental results. In particular it predicts lower value for Neel temperature $\left(T_{1}\right)$, i.e. the temperature at which spontaneous magnetization of subllatice becomes zero. Fig.5 also indicates the existence of the re-entrant phase between $T_{1}$ and $T_{2}$. Note that for sufficiently strong background disorder, the measure of which is the ground state entropy, the temperature $T_{2}$ may be equal to zero as in antiferromagnetic Ising model on two dimensional triangular lattice [1, 5].

Peculiar result of our calculation is the range of temperature in between $T_{1}$ and $T_{2}$, where we have continuous phase transition at a fixed magnetic field. This is an example of Griffiths singularities well known in glassy systems [6, 7].

Recently we obtain similar singularities in three-site interacting Ising model on Husimi tree, where magnetization exhibits chaotic size dependence 8]. In three-site interacting Ising model we considered the Lyapunov exponents as an order parameter and obtained the scaling in terms of Lyapunov exponents 9, 10, 11.

In the presence of Griffiths singularities the scaling relation between spontaneous magnetization $m_{0}$ and reduced temperature $t=\left(T-T_{1}\right) / T$ is well known relation

$$
m_{0} \sim(-t)^{\beta}
$$

when we approach to $T_{1}$ from low temperature. But the magnetization has the following non analytic dependence on magnetic field in the temperature range from $T_{1}$ to $T_{2}$ :

$$
m \sim t^{\Phi} h^{2 t}|\ln h| .
$$


For dilute spin glass $\Phi=1+\beta[$ []].

It will be very interesting to calculate the values of critical exponents $\beta$ and $\Phi$ in our case. As to the presence of the ordered state at finite temperature, one can recall another well known model with macroscopically degenerate ground state - antiferromagnetic Potts model. It has been shown in number of work that in some dimensions antiferromagnetic Potts model can have distinct low-temperature ordered phase with algebraically decaying correlations [12]. On the Bethe and Bethe-like lattice the dimensionality effects can be simulated by changing the coordination numbers. How this will affect on phase structure we are also going to find out in our farther work.

This work was partly supported by the Grant-211-5291 YPI of the German Bundesministerium fur Forshung and Technologie and by the Grant INTAS-96-690. 


\section{References}

[1] R. Liebmann in Lecture Notes in Physics Vol. 251, Springer-Verlag, Berlin (1986).

[2] M.F. Collins and O.A. Petrenko, "Triangular Antiferromagnets", [condmat/9706153.

[3] J. L. Monroe, J. Stat. Phys. 65, 255 (1991); ibid 67, 1185 (1992).

[4] Ananikian N.S. Akheyan A.Z. Zh. Eksp.Teor.Fiz. 107 (1995) 196-208;Akheyan A.Z. Ananikian N.S. J.Phys.A 29 (1996), 721-731.

[5] J. Doczi-Reger and P.C. Hemmer, Physica A 108, 531 (1981)

[6] R.B. Griffiths, Phys.Rev.Lett. 23, 17 (1969).

[7] B.K. Chakrabarti, A. Dutta and P. Sen in Lecture Notes in Physics Vol. m 41, Springer-Verlag, Berlin (1996).

[8] A.E. Alahverdian, N.S. Ananikian and S.K. Dallakian, "Singularites at a Dense Set of Temperature in Husimi Tree ", cond-mat/9702106

[9] N.S. Ananikian, S.K. Dallakian,N.Sh. Izmailian and K.A. Oganessyan, Phys. Lett. A 214, 205 (1996); Erratum 221, 434 (1996)

[10] N.S. Ananikian, S.K. Dallakian,N.Sh. Izmailian and K.A. Oganessyan, Fractals 5, 175 (1997).

[11] N.S. Ananikian and S.K. Dallakian, Physica D 107, 75 (1997).

[12] Racz Z. Vicsek T. Phys.Rev.B 27 (1983) 2992. 


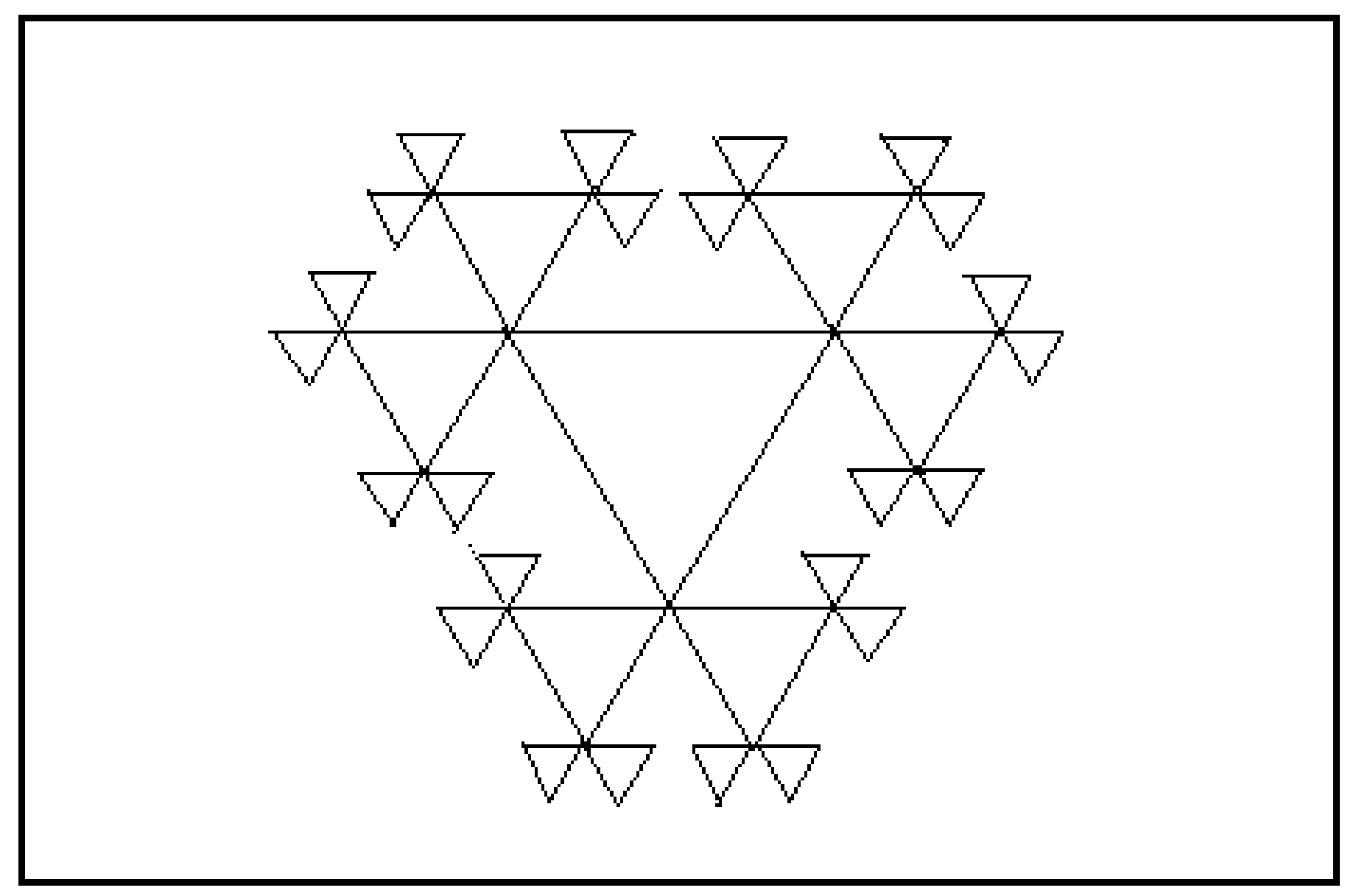

Figure 1: Husimi tree with $\gamma=3$ 


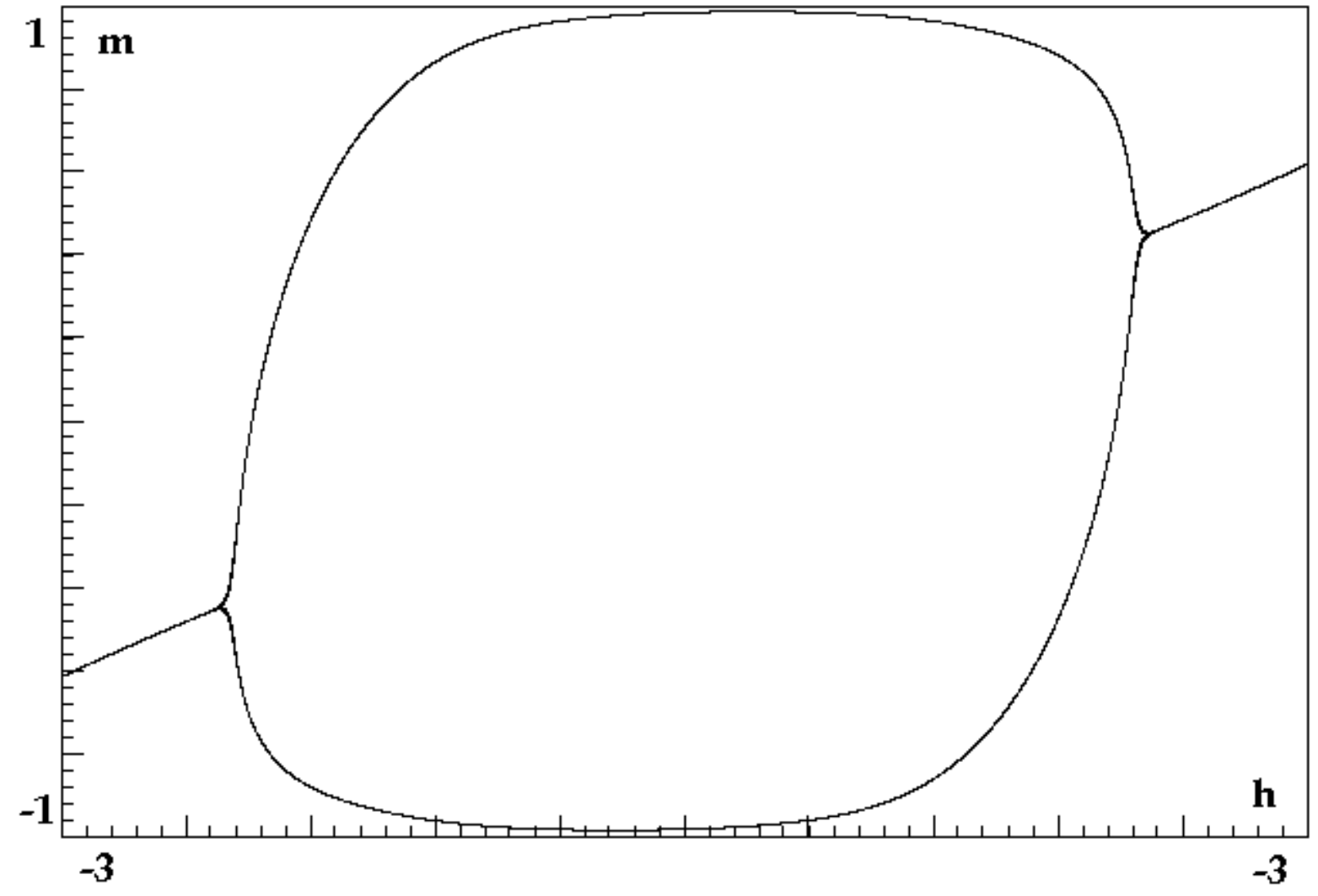

Figure 2: Plot of $m$ versus $h(J=-0.5, \gamma=3)$ 


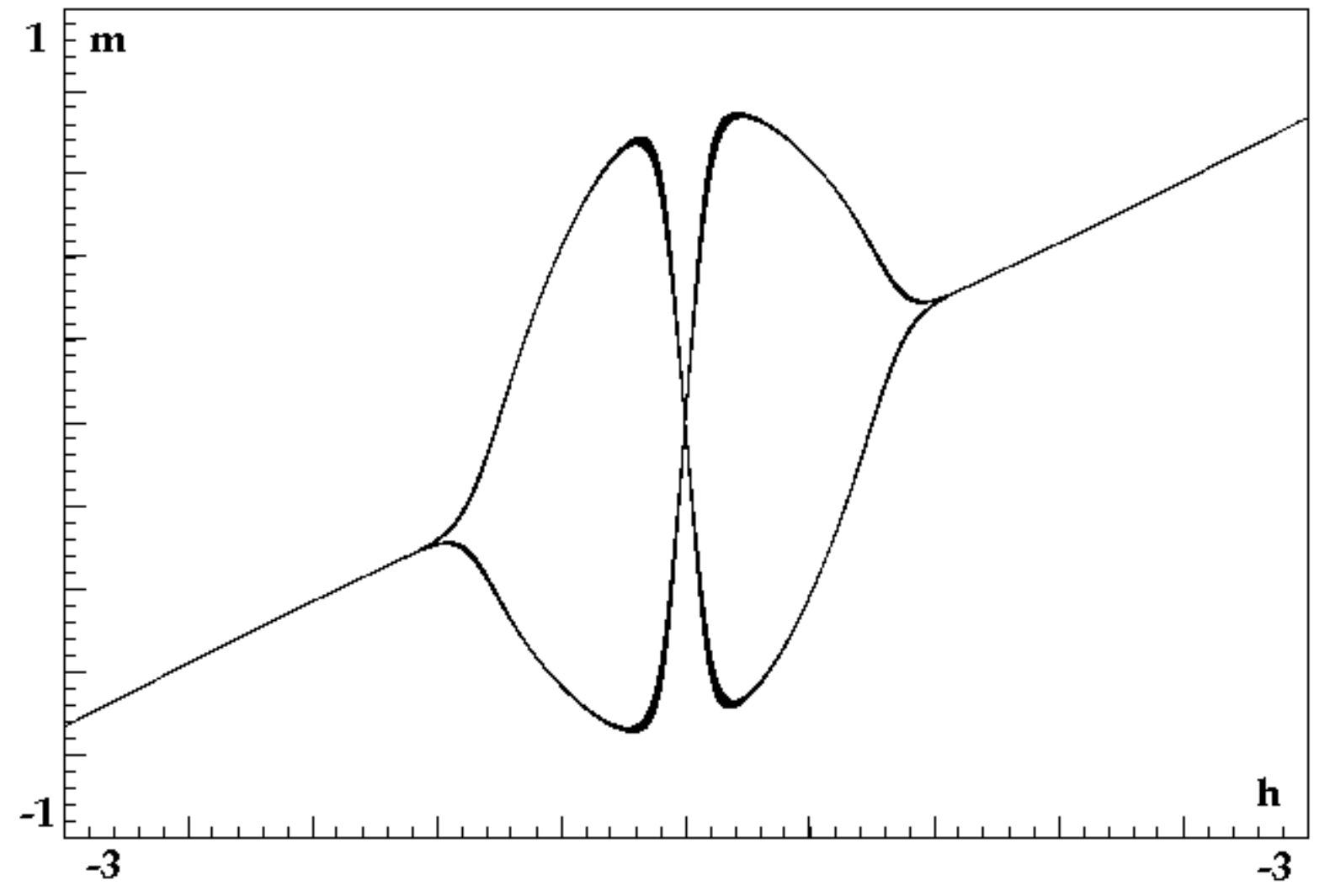

Figure 3: Plot of $m$ versus $h(J=-0.41, \gamma=3)$ 


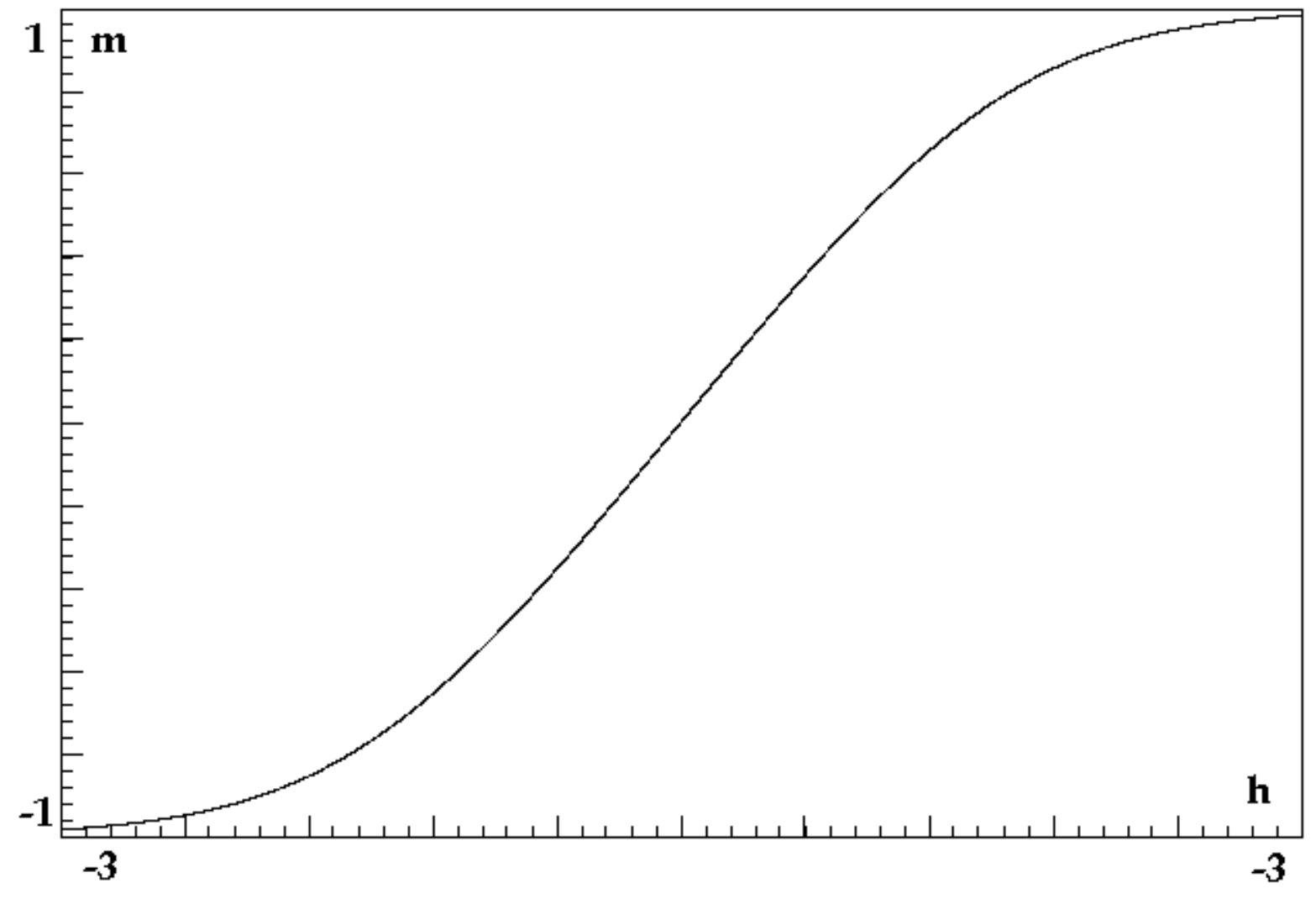

Figure 4: Plot of $m$ versus $h(J=-0.1, \gamma=3)$ 


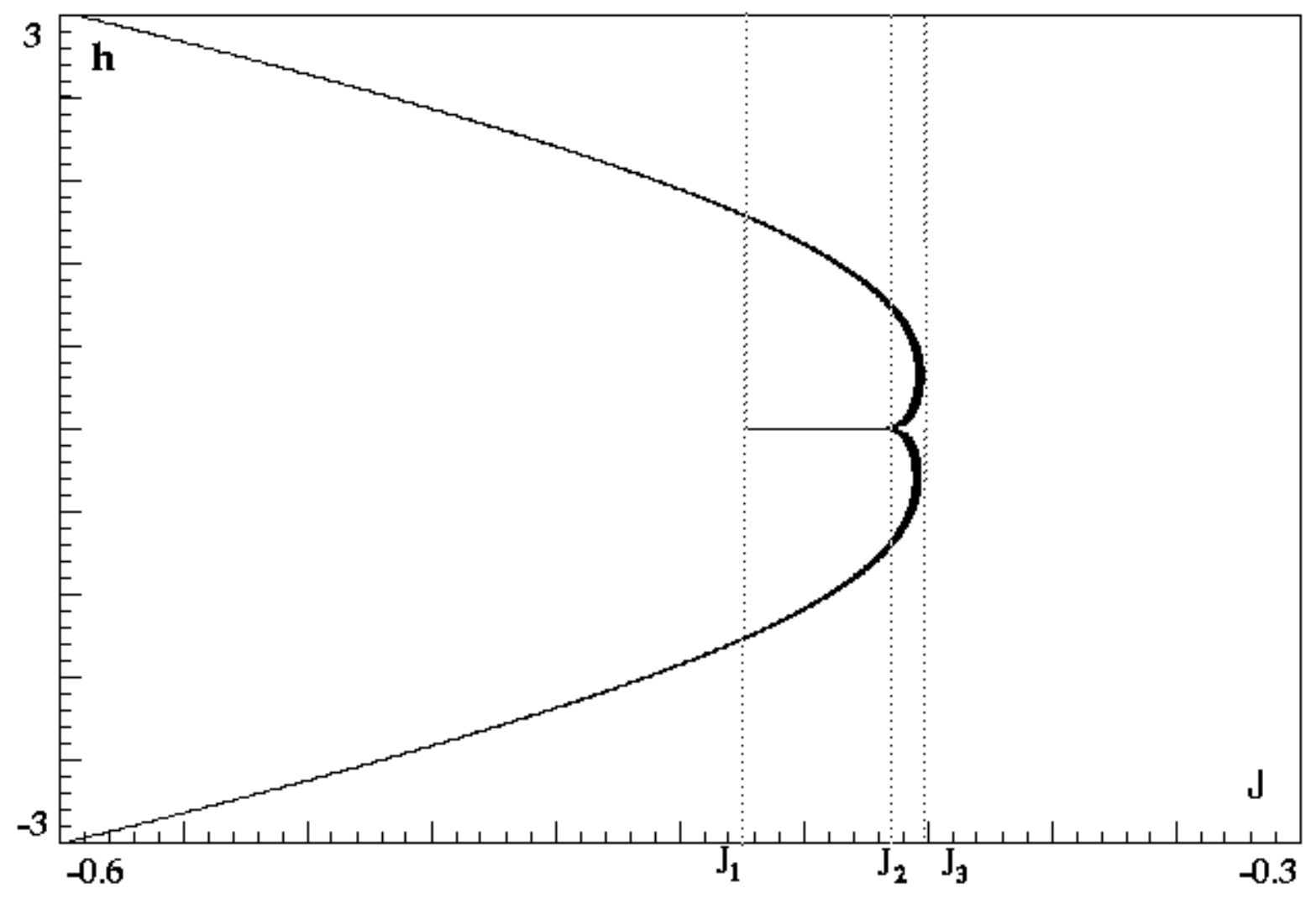

Figure 5: The phase diagramm of antiferromagnetic Ising model in Husimi tree $(\gamma=3)$. 